\title{
Inactivation of the Prelimbic Cortex Attenuates Context-Dependent Operant Responding
}

\author{
(D)Sydney Trask, Megan L. Shipman, John T. Green, and Mark E. Bouton \\ University of Vermont, Burlington, Vermont 05405-0134
}

\begin{abstract}
Operant responding in rats provides an analog to voluntary behavior in humans and is used to study maladaptive behaviors, such as overeating, drug taking, or relapse. In renewal paradigms, extinguished behavior recovers when tested outside the context where extinction was learned. Inactivation of the prelimbic (PL) region of the medial prefrontal cortex by baclofen/muscimol (B/M) during testing attenuates renewal when tested in the original acquisition context after extinction in another context (ABA renewal). Two experiments tested the hypothesis that the PL is important in context-dependent responding learned during conditioning. In the first, rats learned to lever-press for a sucrose-pellet reward. Following acquisition, animals were infused with either B/M or vehicle in the PL and tested in the acquisition context (A) and in a different context (B). All rats showed a decrement in responding when switched from Context A to Context $\mathrm{B}$, but PL inactivation decreased responding only in Context A. Experiment $2 \mathrm{a}$ examined the effects of PL inactivation on ABC renewal in the same rats. Here, following reacquisition of the response, responding was extinguished in a new context $(\mathrm{C})$. Following infusions of $\mathrm{B} / \mathrm{M}$ or vehicle in the $\mathrm{PL}$, responding was tested in Context $\mathrm{C}$ and another new context (D). The rats exhibited ACD renewal regardless of PL inactivation. Experiment $2 \mathrm{~b}$ demonstrated that PL inactivation attenuated the ABA renewal effect in the same animals, replicating earlier results and demonstrating that cannulae were still functional. The results suggest that, rather than attenuating renewal generally, PL inactivation specifically affects ABA renewal by reducing responding in the conditioning context.
\end{abstract}

Key words: context; instrumental learning; operant conditioning; renewal

Significance Statement

Extinguished operant behavior can recover ("renew") when tested outside the extinction context. This suggests that behaviors, such as overeating or drug taking, might be especially prone to relapse following treatment. In rats, inactivation of the prelimbic cortex (PL) attenuates renewal. However, we report that PL inactivation after training attenuates responding in the context in which responding was acquired, but not in another one. A similar inactivation has no impact on renewal when testing occurs in a new, rather than the original, context following extinction. The PL thus has a more specific role in controlling contextually dependent operant behavior than has been previously reported.

\section{Introduction}

The role of the rodent prelimbic (PL) region of the medial prefrontal cortex has been studied in multiple behavioral paradigms that are thought to measure higher-order cognitive function (Kesner and Churchwell, 2011). However, several studies suggest a fundamental role for the PL in supporting

Received Oct. 31, 2016; revised Jan. 23, 2017; accepted Jan. 24, 2017.

Author contributions: S.T., M.L.S., J.T.G., and M.E.B. designed research; S.T. and M.L.S. performed research; S.T. analyzed data; S.T., M.L.S., J.T.G., and M.E.B. wrote the paper.

This work was supported by National Institutes of Health Grant R01 DA033123 to M.E.B., the University of Vermont Neuroscience COBRE facility (National Institutes of Health Grant P30 RR 032135/P30 GM 103498), and the University of Vermont Department of Psychological Science.

The authors declare no competing financial interests.

Correspondence should be addressed to Sydney Trask, Department of Psychological Science, University of Vermont, Burlington, VT 05405-0134. E-mail: sydney.trask@uvm.edu.

DOI:10.1523/JNEUROSCI.3361-16.2017

Copyright $\odot 2017$ the authors $\quad 0270-6474 / 17 / 372317-08 \$ 15.00 / 0$ behavior acquired through operant learning. In the wellknown operant conditioning paradigm, rats learn to perform a response (e.g., lever pressing) that produces a reinforcer (e.g., a food pellet). Responding can then be reduced by removing the reinforcer in a procedure called extinction. Operant responding and its suppression through extinction can provide insight into how voluntary behaviors (e.g., overeating, drug taking) are influenced by reinforcement.

Although extinction reduces responding, it does not erase learning. For example, responding returns when it is tested outside the context (broadly defined as tactile, visual, and olfactory cues that constitute the environment) of extinction. This renewal effect (e.g., Nakajima et al., 2000; Bouton et al., 2011) is especially robust when responding is reinforced in one context (A) extinguished in a second context $(\mathrm{B})$ and then tested in the acquisition context (ABA renewal) (e.g., Nakajima et al., 2000; Nakajima et al., 2002). Renewal also occurs when responding is trained in $A$, 
extinguished in $\mathrm{B}$, and tested in a new context ( $\mathrm{C}$; $\mathrm{ABC}$ renewal), or when responding is acquired and extinguished in $\mathrm{A}$ and tested in a new context (B; $A A B$ renewal) (Bouton et al., 2011; Todd, 2013). ABC and $A A B$ renewal suggests that renewal can be caused by mere removal from the extinction context. However, ABA renewal is often stronger than $\mathrm{ABC}$ and $\mathrm{AAB}$ (Bouton et al., 2011), suggesting that returning to the original context is especially effective at restoring behavior.

Operant responding itself is at least partly context-specific. That is, following acquisition, a context change results in a decrement in responding (Bouton et al., 2011, 2014; Thrailkill and Bouton, 2015). This suggests that during acquisition, the context is encoded as part of the structure that underlies operant learning. The context (S) could enter into a number of associations with the response $(\mathrm{R})$ and/or outcome $(\mathrm{O})$, including a direct link with the response (S-R) (Thrailkill and Bouton, 2015), or a more "hierarchical" link with the response-outcome association, $\mathrm{S}-(\mathrm{R}-\mathrm{O})$ (Trask and Bouton, 2014).

Recent research has investigated the neural mechanisms supporting renewal after operant extinction. Studies have implicated the PL in the ABA renewal of cocaine-reinforced (Fuchs et al., 2005) and alcohol-reinforced (Willcocks and McNally, 2013) responding (but see Bossert et al., 2011, where infralimbic cortex, rather than $\mathrm{PL}$, is implicated in ABA renewal of heroin-reinforced responding). In related experiments, Eddy et al. (2016) trained rats to lever press for a sucrose reward in Context A and then extinguished responding in Context B. Before testing in both Contexts $A$ and $B$, half the rats received infusions of $\mathrm{GABA}(\mathrm{A} / \mathrm{B})$ receptor agonists baclofen and muscimol into the PL (temporarily inactivating the region), and half received vehicle infusions. Although groups showed no difference in responding in the extinction context, animals with the inactivated PL showed attenuated renewal in Context A. Eddy et al. (2016) proposed that rather than increasing transfer of inhibition learned in extinction across contexts, PL inactivation caused a decrease of excitatory operant responding in the acquisition context. In the present experiments, we therefore hypothesized that PL inactivation attenuates ABA renewal by reducing context-dependent operant behavior.

\section{Materials and Methods}

In Experiment 1, lever pressing produced sucrose in one context, Context A. Extinction tests then occurred in Context A and Context B, a new context. PL inactivation during testing decreased responding in the acquisition context, but not Context B. Experiment 2a then found that PL inactivation did not affect $A B C$ renewal, whereas Experiment $2 b$ confirmed that PL inactivation did attenuate ABA renewal (when testing occurred in the acquisition context). These results suggest that the PL specifically supports operant responding in the context where it is learned, and this is the mechanism through which PL inactivation reduces ABA renewal.

\section{Experiment 1}

The purpose of Experiment 1 was to test the hypothesis that the PL is involved in controlling context-dependent operant responding. Following surgical implantation of guide cannulae into the PL, rats were trained to lever press for a sucrose-pellet reward in Context A. Animals were then tested for responding in the acquisition context and a context in which only magazine training had occurred (B) following either a temporary inactivation of the PL by baclofen/muscimol (B/M) or a saline vehicle infusion to the same region. If, as suggested by Eddy et al. (2016), the weakened ABA renewal effect they observed was due to weakened contextual control by Context $\mathrm{A}$, then responding should be suppressed in Context A following PL inactivation. No such suppression would be expected in Context B, where the operant response had not been trained.
Subjects. The subjects were 24 male Wistar rats purchased from Charles River Laboratories. They were between 59- and 63-d-old at the start of the experiment and were individually housed in a room maintained on a 12:12 h light:dark cycle. Experimentation took place during the light period of the cycle. Following postsurgery recovery, the rats were food-deprived to $90 \%$ of their baseline body weight throughout the experiment.

Surgery. Following acclimation to the colony, rats were anesthetized with isoflurane and stereotaxic surgery was performed to bilaterally implant guide cannulae (26 gauge, Plastics One) in the PL region of the medial prefrontal cortex ( $\mathrm{mPFC}$ ). Rats were given $5.0 \mathrm{mg} / \mathrm{kg}$ of carprofen for analgesia both during surgery and $1 \mathrm{~d}$ postoperatively. During surgery, bupivacaine was also administered as a local anesthetic $(0.15 \mathrm{ml})$ and $1 \mathrm{ml}$ of lactated Ringer's solution was administered for hydration. Coordinates used were $3.0 \mathrm{~mm}$ from bregma, $\pm 0.75 \mathrm{~mm}$ from midline, and $-3.0 \mathrm{~mm}$ ventral from bregma. Following surgery, rats were given 5-6 d of recovery. After recovery, a new baseline weight was taken and rats began food deprivation.

Apparatus. Two sets of four conditioning chambers that were housed in separate rooms of the laboratory served as the two contexts (counterbalanced). Each chamber was housed in its own sound attenuation chamber. All boxes were of the same design (Med Associates model ENV008 -VP). They measured $30.5 \times 24.1 \times 21.0 \mathrm{~cm}(1 \times \mathrm{w} \times \mathrm{h})$. A recessed $5.1 \mathrm{~cm} \times 5.1 \mathrm{~cm}$ food cup was centered in the front wall $\sim 2.5 \mathrm{~cm}$ above the level of the floor. A retractable lever (Med Associates model ENV$112 \mathrm{CM}$ ) positioned to the left of the food cup protruded $1.9 \mathrm{~cm}$ into the chamber. The chambers were illuminated by one $7.5 \mathrm{~W}$ incandescent bulb mounted to the ceiling of the sound attenuation chamber, $\sim 34.9 \mathrm{~cm}$ from the grid floor at the front wall of the chamber. Ventilation fans provided background noise of $65 \mathrm{dBA}$.

In one set of boxes, the side walls and ceiling were made of clear acrylic plastic, whereas the front and rear walls were made of brushed aluminum. The floor was made of stainless steel grids $(0.48 \mathrm{~cm}$ diameter $)$ staggered such that odd- and even-numbered grids were mounted in two separate planes, one $0.5 \mathrm{~cm}$ above the other. This set of boxes had no distinctive visual cues on the walls or ceilings of the chambers. A dish containing $5 \mathrm{ml}$ of lemon cleaner (Rite Aid) was placed outside of each chamber near the front wall.

The second set of boxes was similar to the lemon-scented boxes, except for the following features. In each box, one side wall had black diagonal stripes, $3.8 \mathrm{~cm}$ wide and $3.8 \mathrm{~cm}$ apart. The ceiling had similarly spaced stripes oriented in the same direction. The grids of the floor were mounted on the same plane and were spaced $1.6 \mathrm{~cm}$ apart (center-tocenter). A distinct odor was continuously presented by placing $5 \mathrm{ml}$ of Pine-Sol (Clorox) in a dish outside the chamber.

The reinforcer was a $45 \mathrm{mg}$ sucrose-based food pellet (5-TUT: 1811251, TestDiet) delivered to the magazine. The apparatus was controlled by computer equipment located in an adjacent room.

Procedure: magazine training. On the first day of the experiment, all rats were assigned to a box within each set of chambers. They then received one $30 \mathrm{~min}$ session of magazine training in one box. On the same day, the animals also received a second $30 \mathrm{~min}$ session of magazine training in the other box. Half the animals were trained first in the box later used as Context A, and half were trained first in the box used as Context $B$. The sessions were separated by $\sim 1 \mathrm{~h}$. Once all animals were placed in their respective chambers, a 2 min delay was imposed before the start of the session. In each magazine training session, $\sim 60$ reinforcers were delivered freely on a random time 30 s (RT 30 s) schedule. The levers were not present during this training.

Procedure: acquisition. On each of the next $6 \mathrm{~d}$, all rats received one 30 min session of instrumental training in Context A. Following a 2 min delay after the rat was placed in the chamber, sessions were initiated by the insertion of the lever into the chamber. Throughout the sessions, presses on the lever delivered reinforcers on a variable interval $30 \mathrm{~s}$ (VI $30 \mathrm{~s})$ schedule of reinforcement. No hand shaping was necessary.

Procedure: B/M infusions. On the final day of the experiment, rats were given an infusion via Hamilton syringes of either $0.9 \%$ saline vehicle (control) or B/M (1.0 mm/0.1 mm; Sigma-Aldrich) dissolved in $0.9 \%$ saline to temporarily inactivate the PL region. Internal cannulae (33 
gauge, Plastics One) were inserted bilaterally into guide cannulae. Internal cannulae tips protruded $1 \mathrm{~mm}$ below the guide cannulae tip. An infusion of $0.5 \mu \mathrm{l}$ per side was delivered at a rate of $0.25 \mu \mathrm{l}$ per minute using a microinfusion pump. Following completion of the infusion, the internal cannulae were left in place for $1 \mathrm{~min}$ to allow diffusion of the drug or saline away from the cannulae tips. Internal cannulae were then removed and dummy cannulae replaced. Each rat was then placed in a transportation container. Time between the end of infusion and the start of testing was $\sim 10-30 \mathrm{~min}$.

Test. Following infusions, all rats were given two 10 min extinction tests: one in Context A and one in Context B. As usual, following a 2 min delay, each test session began with the insertion of the lever and ended with its retraction. Testing order was counterbalanced such that half the animals in each group (B/M or saline infusion) were tested first in Context $\mathrm{A}$, and half of the animals were tested first in Context $\mathrm{B}$. There was a delay of $\sim 30$ min between tests for each animal.

\section{Experiment $2 a$}

If it is true that PL inactivation specifically affects responding in the conditioning context (Experiment 1), then other forms of renewal that do not involve a return to the context of acquisition (e.g., $\mathrm{ABC}$ and $\mathrm{AAB}$ renewal) should be unaffected by inactivation of the PL. To test this hypothesis, all animals from Experiment 1 were given further training back in Context A to lever press for the sucrose reinforcer. They were then switched to a novel context (Context C) where responding no longer produced the reinforcer. Once responding was extinguished, the rats were then given infusions of either $\mathrm{B} / \mathrm{M}$ or saline vehicle to the PL and tested (in a counterbalanced order) in the extinction context (Context C) as well a novel context (Context D). It was predicted that, when animals were tested outside of both the acquisition and extinction contexts (i.e., in Context D), we would observe an $\mathrm{ABC}$ (or “ACD") renewal effect that would be unaffected by PL inactivation as no contextual control by Context D was expected.

Subjects. The subjects were the same animals used in Experiment 1, housed and maintained in the same way.

Apparatus. The same apparatus was used as Experiment 1. However, two additional sets of four conditioning chambers housed in separate rooms of the laboratory served as the two new contexts (Contexts $\mathrm{C}$ and $\mathrm{D}$, counterbalanced). Each chamber was housed in its own sound attenuation chamber. All boxes were of the same design as before (Med Associates model ENV-008-VP) and had the same general features as the chambers described in Experiment 1.

The new boxes differed as follows to serve as the different contexts. In one set, the side walls and ceiling were made of clear acrylic plastic, whereas the front and rear walls were made of brushed aluminum. There was a $1.5 \mathrm{~cm}$ vertical gray stripe down the center of one acrylic side wall. In this set of boxes, the grids of the floor were spaced $1.6 \mathrm{~cm}$ apart (center-to-center). A distinct odor was continuously presented by placing $5 \mathrm{ml}$ of white vinegar (Price Chopper) in a dish outside the chamber. The second set of boxes had no adornment of the side walls. The floor consisted of alternating stainless steel grids with different diameters $(0.5$ and $1.3 \mathrm{~cm}$, spaced $1.6 \mathrm{~cm}$ apart). A distinct odor was continuously presented by placing $\sim 1$ g of Vick's Vaporub (Procter \& Gamble) in a dish outside the chamber. The reinforcer was the same sucrose-based food pellet used in Experiment 1.

Procedure: magazine training. On the first day of this experiment, all rats were assigned to a box within each set of new chambers. They then received one $30 \mathrm{~min}$ session of magazine training in the chamber that became Context C. On the same day, the animals also received a second 30 min session of magazine training in the chamber that would serve as Context D. Half the animals were trained first in Context $C$, and half were trained first in Context D. The sessions were separated by $\sim 1 \mathrm{~h}$. The reinforcers were delivered exactly as in Experiment 1.

Procedure: acquisition. On each of the next $6 \mathrm{~d}$, all rats received one 30 min session of instrumental training in their original Context A (from Experiment 1). These sessions proceeded in exactly the same way as Experiment 1.

Procedure: extinction. On each of the next $4 \mathrm{~d}$, all animals then received one session of extinction in Context C. As before, following a 2 min delay, the lever was inserted and available for $30 \mathrm{~min}$. During this phase, responding on the lever had no programmed consequences.

Procedure: infusions. On the final day of the experiment, all animals received an infusion of either $0.9 \%$ saline vehicle or $\mathrm{B} / \mathrm{M}$ dissolved into $0.9 \%$ saline vehicle as before. Infusions proceeded similarly to Experiment 1 , with the sole exception being that half the animals that previously received a saline infusion now received a $\mathrm{B} / \mathrm{M}$ infusion and half received the saline infusion. The same was true of animals that had previously received the $\mathrm{B} / \mathrm{M}$ infusion: half now received a saline infusion and half now received the $\mathrm{B} / \mathrm{M}$ infusion. Time between the end of infusion and the start of the first test was $\sim 10-45 \mathrm{~min}$.

Test. Following infusions, all rats were given two 10 min extinction tests: one in Context C and one in Context D. Following a 2 min delay, each test session began with the insertion of the lever and ended with the retraction of the lever. Testing order was counterbalanced such that half the animals were tested first in Context $\mathrm{C}$, and half were tested first in Context D. As in Experiment 1, tests were separated by $\sim 30 \mathrm{~min}$.

\section{Experiment $2 b$}

Although our hypothesis predicted the null between-groups effect observed in Experiment 2a, the presence of a null effect leaves the results open to other possible interpretations. For example, it is possible that the cannulae were no longer properly functioning by the time of the renewal test, or perhaps there had been receptor desensitization or downregulation. To test for such possibilities, all animals were returned to Context $\mathrm{A}$ for a single reacquisition session in which lever pressing produced the sucrose-pellet reward, followed by extinction in Context B in which the lever press no long produced the reinforcer. Animals were then tested for responding in both Context $\mathrm{A}$ and Context $\mathrm{B}$ after receiving the same infusion they received in Experiment 2a. We hypothesized that, as in Eddy et al. (2016), the rats would show an attenuated ABA renewal effect following PL inactivation. If that result was obtained, the possibility that the null effect observed in Experiment $2 \mathrm{a}$ was due to malfunctioning cannulae or decreased drug potency could be reasonably excluded.

Subjects. The subjects were the same rats used in Experiment 1 and Experiment 2a, housed and maintained in the same way.

Apparatus. The apparatus was the same as the one used in Experiment 1. All animals were assigned the same Context A (lemon-scented chambers or pine-scented chambers, counterbalanced) and Context B (pinescented chambers or lemon-scented chambers, counterbalanced) as they had been in Experiment 1 .

Procedure: acquisition. At $48 \mathrm{~h}$ following the preceding renewal test, the rats were given one session in which lever presses were reinforced with sucrose pellets in Context A following the procedures described above.

Procedure: extinction. On each of the next $2 \mathrm{~d}$, all animals then received two sessions of extinction of responding in Context B (Context B was the same Context B for each animal from Experiment 1, counterbalanced as the lemon-scented boxes or the pine-scented boxes). The same extinction procedures described above (Experiment 2a) were used.

Procedure: infusions. On the final day of the experiment, all animals received an infusion of either $0.9 \%$ saline vehicle or $\mathrm{B} / \mathrm{M}$ dissolved into $0.9 \%$ saline vehicle as before. Infusions proceeded exactly as they had in Experiment 2a. The same rats that received B/M infusions in Experiment 2a received $\mathrm{B} / \mathrm{M}$ infusions here. The same was true of animals that had received vehicle infusions in Experiment 2a.

Test. Following infusions, all rats were given two 10 min extinction tests: one in Context A and one in Context B. Following a 2 min delay, each test session began with the insertion of the lever and ended with the retraction of the lever. Testing order was counterbalanced such that half the animals were tested first in Context $\mathrm{A}$, and half were tested first in Context B. Sessions were separated by $\sim 30 \mathrm{~min}$.

\section{Results}

\section{Experiment 1}

Data analysis

All data were subject to ANOVA or $t$ tests where appropriate. The rejection criterion was set to $p<0.05$. 


\section{Histology}

Following the final test (see Materials and Methods; Experiment $2 \mathrm{~b}$ ), rats were injected with a lethal dose of sodium pentobarbital $(150 \mathrm{mg} / \mathrm{kg}$, i.p.) and then transcardially perfused with $0.9 \%$ saline and $10 \%$ buffered formalin. Brains were removed and stored in $10 \%$ formalin. Five to $7 \mathrm{~d}$ before embedding in an optimal cutting temperature compound, brains were transferred to a $30 \%$ sucrose $/ 10 \%$ buffered formalin solution for cryoprotection. After being frozen in optimal cutting temperature compound, tissue was sectioned at $60 \mu \mathrm{m}$ intervals with a cryostat. Sections were then mounted onto chrome alum subbed slides where they dried before staining. Tissue was stained with cresyl violet and allowed to dry before being glass coverslipped with Permount. Cannula placement was verified by examining the lowest part of the cannula tracts (and adding $1 \mathrm{ml}$ for the site of infusion) and compared with a rat brain atlas (for verified cannula placements and a representative image of the cannula track, see Fig. 1) (Paxinos and Watson, 2007). Six animals were excluded due to failure to verify one or both cannulae placements. This resulted in $n$ values of 9 for both groups in Experiment 1 and $n=7$ for Group B/M and $n=11$ for Group Vehicle in Experiments 2a and $2 b$.

\section{Acquisition}

As expected, all animals increased their responding throughout acquisition (Fig. 2A). This was confirmed by a 2 (Group: B/M vs Vehicle) $\times 6$ (Session) ANOVA, which found a main effect of $\operatorname{session}\left(F_{(5,80)}=131.99, \mathrm{MSE}=16.46, p<0.001, \eta_{p}^{2}=0.90\right)$ but no main effect of group $\left(F_{(1,16)}=1.59, \mathrm{MSE}=16.46, p>0.05\right)$ or a significant interaction $(F<1)$.

\section{Test}

During the test (Fig. $2 B$ ), animals that had received the $\mathrm{B} / \mathrm{M}$ infusion responded significantly less than animals that received the saline infusion in Context A, but not Context B. This was confirmed by a 2 (Context: A vs B) $\times 2$ (Group: B/M vs Vehicle) ANOVA, which found a significant main effect of context $\left(F_{(1,16)}=48.84, \operatorname{MSE}=5.23, p<0.001, \eta_{p}^{2}=0.75\right)$, a significant main effect of group $\left(F_{(1,16)}=5.10\right.$, MSE $=26.63$, $p<0.05, \eta_{p}^{2}=0.24$ ), and (most important) a significant Group $\times$ Context interaction $\left(F_{(1,16)}=5.75, \mathrm{MSE}=5.79, p<\right.$ $\left.0.05, \eta_{p}^{2}=0.26\right)$. Follow-up comparisons showed that the groups differed in Context $\mathrm{A}\left(F_{(1,16)}=6.14, p<0.05, \eta_{p}^{2}=0.28\right)$, but not in Context $\mathrm{B}\left(F_{(1,16)}=2.39, p>0.05\right)$. Additionally, both groups showed a significant decrement in responding in Context $\mathrm{B}$ compared with Context A (B/M: $F_{(1,16)}=10.54, p<0.01, \eta_{p}^{2}=0.40$; Vehicle: $\left.F_{(1,16)}=44.05, p<0.001, \eta_{p}^{2}=0.73\right)$. A $t$ test conducted to compare responding of $\mathrm{B} / \mathrm{M}$ animals in Context A relative to Vehicle animals in $\mathrm{B}$ found that responding was similar in these two conditions $(t(16)=0.13, p=0.99)$. Thus, inactivation of the PL had an effect on responding that was similar to that of changing the context. Further, animals whose cannulae could not be localized to the PL performed almost identically to controls (Context A: mean, 15.15; Context B: mean, 7.50). There were 6 such animals ( $3 \mathrm{~B} / \mathrm{M}$ and $3 \mathrm{Veh}$ ); rats with misplaced cannulae that received the $\mathrm{B} / \mathrm{M}$ infusion had means of 14.87 in Context $\mathrm{A}$ and 4.83 in Context B. Following inactivation of the PL, animals showed suppressed responding in Context A relative to animals that did not receive the inactivation. This effect was not observed in Context B, where the response had not been trained. Thus, inactivation of the PL had a specific impact on responding in the context in which the response had produced the reinforcer.
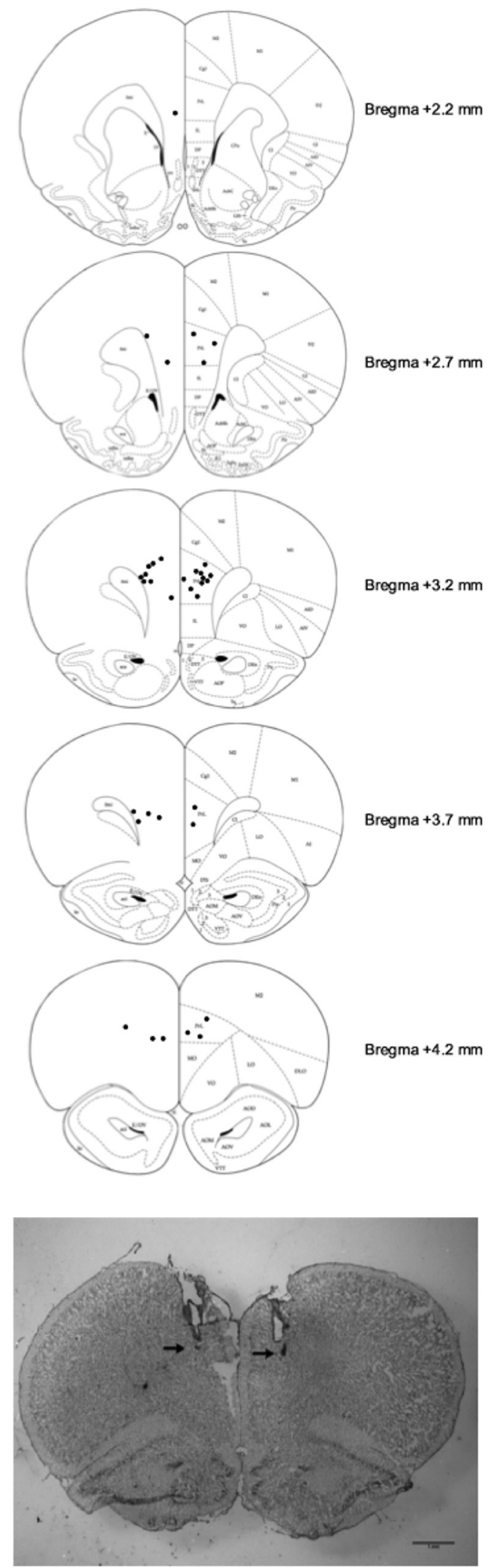

Figure 1. Cannulae tip placements in PL cortex as verified on Nissl-stained sections. Modified with permission from Paxinos and Watson (2007). Bottom, Representative image of the bilateral cannulae tracks in the PL cortex. Arrows indicate where B/M or vehicle was infused through internal cannulae, which protruded $1 \mathrm{~mm}$ below guide cannulae. 
A

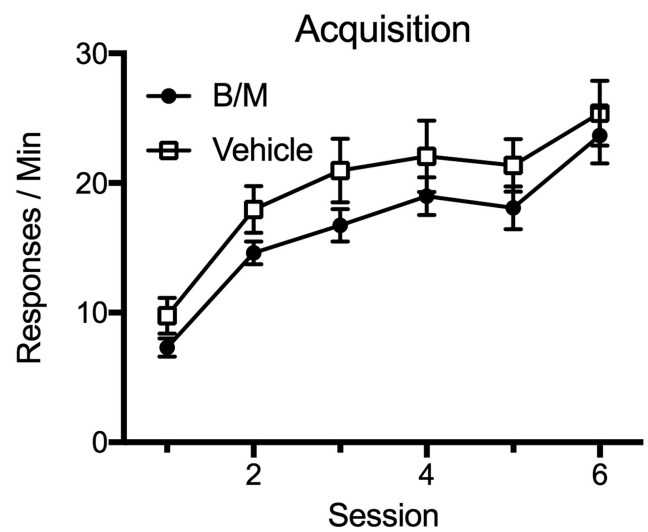

B

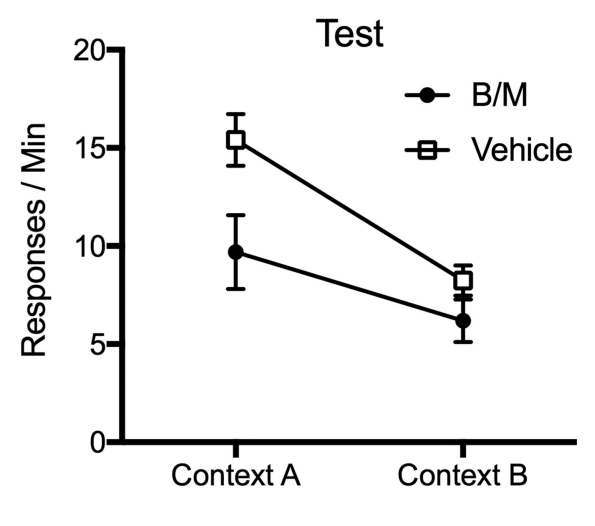

Figure2. Results from acquisition $(\boldsymbol{A})$ and testing $(\boldsymbol{B})$ in Experiment 1. Error bars indicate SEM. There are changes in yaxes. Although groups responded similarly during acquisition, PL inactivation before testing attenuated responding in Context $A$ (where responding had been trained), but not Context $B$ (where responding had not been trained).
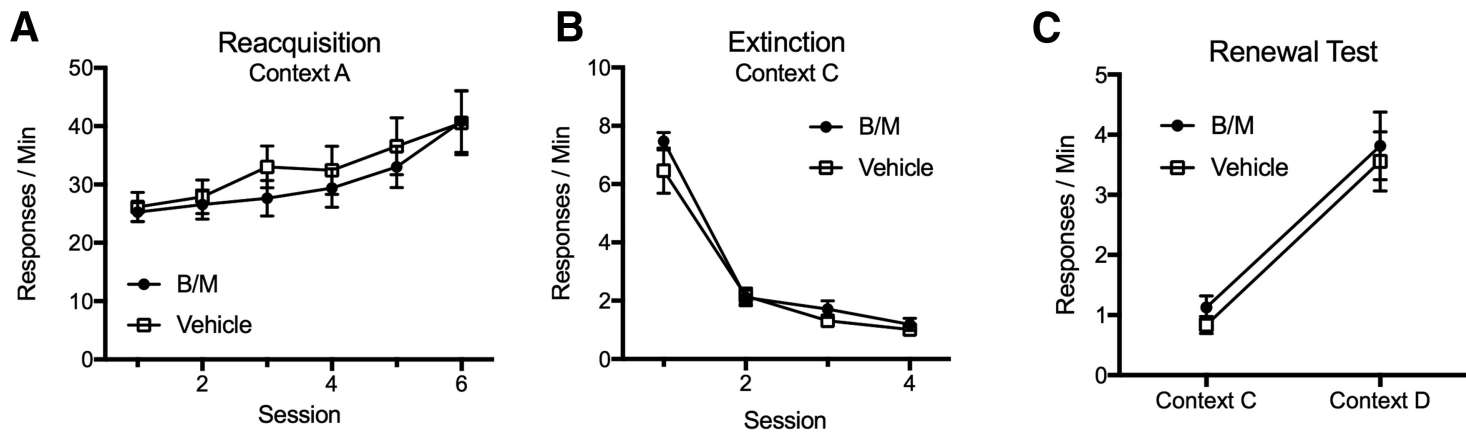

Figure 3. Results from acquisition $(\boldsymbol{A})$, extinction $(\boldsymbol{B})$, and renewal testing $(\boldsymbol{C})$ in Experiment 2a. Error bars indicate SEM. There are changes in $y$ axes. Groups did not differ in acquisition or extinction. PL inactivation before testing had no impact on renewal in a context where responding had not been trained, which was robust and similar between groups.

\section{Experiment 2a}

Acquisition

The increase in responding throughout acquisition in Context $\mathrm{A}$ (Fig. 3A) was confirmed by a 2 (Group: B/M vs Vehicle) $\times 6$ (Session) ANOVA, which revealed a main effect of session $\left(F_{(5,80)}=20.46, \mathrm{MSE}=24.89, p<0.001, \eta_{p}^{2}=0.56\right)$, but neither a main effect of group nor a group $\times$ session interaction $(F$ values $<1)$.

\section{Extinction}

In extinction (Fig. 3B) in Context C, all animals decreased their responding similarly. This was confirmed by a 2 (Group: $\mathrm{B} / \mathrm{M}$ vs Vehicle) $\times 4$ (Session) ANOVA, which found a main effect of session $\left(F_{(3,48)}=118.27, \mathrm{MSE}=1.07, p<0.001, \eta_{p}^{2}=\right.$ $0.84)$ but no main effect of group or an interaction between the $2(F$ values $<1)$.

Test

During the tests in Context C and Context D (Fig. 3C), all animals showed an increase in responding (renewal) in Context D. This was confirmed by a 2 (Context: $\mathrm{C}$ vs $\mathrm{D}) \times 2$ (Group: B/M vs Vehicle) ANOVA, which found a main effect of session $\left(F_{(1,16)}=\right.$ $\left.53.61, \mathrm{MSE}=1.17, p<0.001, \eta_{p}^{2}=0.77\right)$ but no main effect of group and no group $\times$ context interaction $(F$ values $<1)$. As in Experiment 1, the 6 animals whose cannulae could not be localized to the PL performed similarly to the controls (Context C: mean, 1.30; Context D: mean, 5.40). For the $5 \mathrm{~B} / \mathrm{M}$ rats with misplaced cannulae, the means were 1.10 in Context $\mathrm{C}$ and 5.10 in Context D. As predicted, animals showed a strong and similar
$\mathrm{ABC}$ renewal effect when tested outside the context of acquisition and extinction. This effect did not differ based on PL inactivation. These results further suggest that inactivation of the PL causes reduced $\mathrm{ABA}$ renewal by specifically weakening the control of responding by Context $\mathrm{A}$.

\section{Experiment 2b}

Acquisition

The rats responded throughout the acquisition session in Context A (Fig. 4A). A $t$ test found no differences between animals that were to receive the $\mathrm{B} / \mathrm{M}$ infusion and animals that were to receive the vehicle infusion $\left(t_{(16)}=0.03, p=0.98\right)$.

\section{Extinction}

All animals similarly decreased their responding during extinction (Fig. $4 A$ ), as confirmed by a 2 (Group: B/M vs Vehicle) $\times 4$ (Session) ANOVA, which found a main effect of session $\left(F_{(3,48)}=\right.$ 22.86, MSE $\left.=0.31, p<0.001, \eta_{p}^{2}=0.58\right)$, but neither a main effect of group $\left(F_{(1,16)}=1.40\right.$, MSE $\left.=0.48, p>0.05\right)$ nor an interaction $(F<1)$. Given that the animals had previously received extinction in Context B, it was not surprising to see relatively little responding in that context (thanks to contextual cueing) even at the outset of the extinction phase.

\section{Test}

Data from the test are shown in Figure $4 B$. A 2 (Context) $\times 2$ (Group: B/M vs Vehicle) ANOVA was run to assess responding during the test. This revealed a significant effect of context $\left(F_{(1,16)}=36.81, \mathrm{MSE}=19.81, p<0.001, \eta_{p}^{2}=0.70\right)$ but no main 
effect of group $(F<1)$. The group $\times$ context interaction approached significance $\left(F_{(1,16)}=3.71, \mathrm{MSE}=19.81, p=0.07\right)$. Given the repeated testing of these animals, a 2 (Context) $\times 2$ (Drug) ANOVA was run to assess responding for the first of the two within-subject tests, effectively creating four testing groups (Context A: B/M; Context A: Vehicle; Context B: B/M; and Context B: Vehicle). This revealed a significant main effect of context $\left(F_{(1,14)}=13.77, \mathrm{MSE}=27.56, p<0.01\right.$, $\left.\eta_{p}^{2}=0.50\right)$ and no main effect of drug $\left(F_{(1,14)}=4.82, \mathrm{MSE}=27.55, p<0.05, \eta_{p}^{2}\right.$ $=0.26)$. The interaction between the two was significant $\left(F_{(1,14)}=4.82\right.$, MSE = 27.55, $\left.p<0.05, \eta_{p}^{2}=0.26\right)$. Follow-up comparisons showed Group Vehicle $\left(F_{(1,14)} \mathrm{MSE}=22.60, p<\right.$ $\left.0.001, \eta_{p}^{2}=0.62\right)$ responded more in Context A than Context $\mathrm{B}$ (but not Group B/M, $F<1$ ). Additional comparisons showed that, although Groups B/M and Vehicle performed similarly in Context B $(F<1)$, Group B/M was significantly suppressed relative to Group Vehicle in Context $\mathrm{A}\left(F_{(1,14)}=9.16, \mathrm{MSE}=27.55\right.$, $\left.p<0.01, \eta_{p}^{2}=0.40\right)$. Animals whose cannulae could not be localized to the PL ( $n=6 ; 3$ Context A B/M, 2 Context B B/M, 0 Context $\mathrm{A}$ Veh, and 1 Context $\mathrm{A}$ Veh) again performed similarly to controls (Context A, mean, 15.30; Context B, mean, 2.20). When isolating only the animals with misplaced cannulae who received $\mathrm{B} / \mathrm{M}$ infusions, the means were 15.3 in Context $\mathrm{A}$ and 2.45 in Context B. As predicted, PL inactivation attenuated the ABA renewal effect. This replicates previous findings (see also Fuchs et al., 2005; Willcocks and McNally, 2013; Eddy et al., 2016) and suggests that the $\mathrm{B} / \mathrm{M}$ infusion and cannulae were still functional during the previous $\mathrm{ABC}(\mathrm{ACD})$ renewal test.

\section{Discussion}

The current results demonstrate that PL inactivation suppresses operant responding when animals are tested in the original acquisition context, but not in a new context (Experiment 1). Further, responding in Context A by the group with the inactivated PL resembled that of the vehicle animals in Context $\mathrm{B}$. The pattern clearly suggests that $\mathrm{B} / \mathrm{M}$-induced inactivation of the $\mathrm{PL}$ resulted in weakened context-dependent operant responding. Based on this result, we hypothesized that ABA renewal (as in Eddy et al., 2016) was attenuated by PL inactivation primarily due to weakened context-dependent responding. Lending more support to this, when animals were tested outside of both the context of extinction and the context of acquisition (in an "ABC" renewal design), PL inactivation had no discernible effect on responding, with all animals showing equal and robust renewal in the new context. It is unlikely that this null effect was due to malfunctioning cannulae or drug, given that a subsequent $\mathrm{ABA}$ renewal test revealed that the same infusions into the PL substantially reduced ABA renewal (Experiment $2 \mathrm{~b}$ ). Our results suggest that the likely mechanism through which an inactivated PL attenuates ABA renewal is by weakening responding that is controlled by the acquisition context. To our knowledge, these are the first results demonstrating attenuated responding in the acquisition context following PL inactivation before extinction training. They suggest that the attenuated ABA renewal effect is not dependent on extinction learning and, instead, that the PL may support contextually controlled responding more generally. Further, this is the first demonstration showing that the PL is not needed for expres-

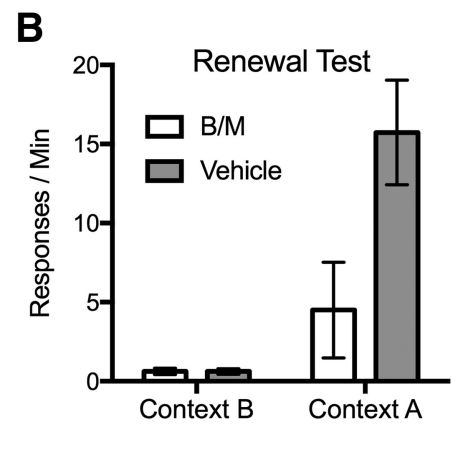

Session

Extinction

Context B

$\rightarrow B / M$

๑ Vehicle

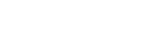
Figure 4. Results from acquisition and extinction $(\boldsymbol{A})$, and renewal testing $(\boldsymbol{B})$ in Experiment $2 \mathrm{~b}$. Error bars indicate SEM. There
are changes in y axes. Although groups responded similarly in acquisition and extinction, PL inactivation before testing attenuated Figure 4. Results from acquisition and extinction $(\boldsymbol{A})$, and renewal testing $(\boldsymbol{B})$ in Experiment $2 \mathrm{~b}$. Error bars indicate SEM. There
are changes in $y$ axes. Although groups responded similarly in acquisition and extinction, PL inactivation before testing attenuated renewal in Context $A$ and had no impact in Context $B$.

sion of $\mathrm{ABC}$ renewal. Given the overall pattern of results, this suggests that $\mathrm{AAB}$ renewal would also be unaffected by inactivation of the PL, as both involve a return of responding outside of the acquisition context. It should be noted, however, that all tests were conducted while the response was being nonreinforced, and that reinforced responding might depend on other mechanisms (different results have been demonstrated examining the effect of the PL on reinforced responding in that there is consistently no detrimental effect of PL lesions or inactivation on it) (Corbit and Balleine, 2003; Jonkman et al., 2009; Willcocks and McNally, 2013). Thus, the PL may have a crucially important role in the expression of nonreinforced responding in the conditioning context.

\section{Alternative interpretations}

At least two caveats about the present results should be noted. First, the results do not necessarily isolate a specific role for the PL over other $\mathrm{mPFC}$ regions. However, the small number of animals given $\mathrm{B} / \mathrm{M}$ infusion through misplaced cannulae never behaved differently from control subjects. And perhaps more important, Experiment 2b's results with ABA renewal directly replicated previous PL results of Eddy et al. (2016). In contrast, Eddy et al. (2016) found that inactivation of the IL increased responding in the extinction context as well as decreased responding in the renewal context; inactivation of MPFC ventral to the IL had no effect. The similarity of the current results to those of the specific results of Eddy et al. (2016) with PL inactivation lends support to the possibility that they were indeed a result of PL inactivation. A second point worth noting is that it is possible that PL inactivation primarily affected higher rates of responding. PL inactivation did not affect responding when responding was relatively low (i.e., in extinction contexts or during renewal in a novel, as opposed to the original, context). Although it is difficult to eliminate a response scale explanation completely, we would note that, in situations where PL inactivation had little effect (Experiment 1's initial test in Context B and Experiment 2a's renewal test in Context D), responding was far from the response floor; the lack of an effect of PL inactivation in these situations was not likely due to a floor effect. However, to rule out a response-rate explanation completely, an experiment would need to confirm that PL inactivation can differentially affect responding in conditions that generate equal levels of responding.

\section{The role of the PL in operant responding}

As noted in the Introduction, other roles for the PL region of the $\mathrm{mPFC}$ have been proposed. For example, in addition to its effects 
on other "higher-order" cognitive functions (e.g., Kesner and Churchwell, 2011), the PL has been implicated in the retrieval of action-outcome associations. In one experiment, Corbit and Balleine (2003) trained rats to perform two responses each for their own distinct outcome (a food pellet or a sucrose solution) following either neurotoxic lesions of the PL or a sham surgery. Once responding was acquired, one of the outcomes was devalued by giving each rat $1 \mathrm{~h}$ of unrestricted access to this food (i.e., through outcome-specific satiation) (Colwill and Rescorla, 1985). Rats were then given access to both responses in extinction. Although no differences were found during acquisition (i.e., both lesion and sham groups performed equally well when reinforced for responding), during the devaluation test animals that had the sham lesion suppressed the response that previously led to the devalued outcome (i.e., expressing goal-directed responding), whereas animals with the lesioned PL suppressed both responses equally. Interestingly, the lesioned group's responses were both suppressed to the level that the sham group responded for the devalued outcome. The authors interpreted this result as evidence that the lesion reduced the animals' ability to retrieve the action-outcome association during extinction. Further consistent with this, when both responses were reinforced following another outcome-specific satiation devaluation procedure, lesioned and nonlesioned subjects performed similarly: in both groups, responding was high for the nondevalued outcome and suppressed for the devalued outcome. Thus, the previous deficit was in the retrieval of the action-outcome association rather than insensitivity to reinforcer devaluation. Further experiments found that other ways to measure knowledge of the action-outcome association (i.e., contingency degradation of one responseoutcome pairing) yielded similar results. That is, when one of two action-outcome contingencies was degraded, animals with PL lesions suppressed responding on both responses equally. Corbit and Balleine (2003) argued that these results further support the claim that the PL is crucial for retrieval of action-outcome associations (see also Balleine and Dickinson, 1998; Killcross and Coutureau, 2003; Coutureau et al., 2009), an interpretation that is perhaps in line with the proposal that the PL has an important role in working memory (Delatour and Gisguet-Verrier, 1996, 1999, 2000; Ragozzino et al., 2002; for review, see Kesner and Churchwell, 2011). The role of the PL also extends to operant reinstatement after extinction (e.g., drug-induced and cueinduced reinstatement) (for review, see Bossert et al., 2013), effects that may depend at least partly on the context (e.g., Baker et al., 1991). Further, and perhaps most pertinent to the present results, Marquis et al. (2007) demonstrated that PL inactivation impaired the ability of the context to guide response choice in a biconditional operant discrimination paradigm. All of these results are compatible with the present findings, which add the suggestion that the suppression of responding observed in PLlesioned subjects should be specific to the context in which it was learned. Thus, all of the research together suggests that the PL is involved in the evocation or excitation of context-dependent operant responding that is based on a learned action-outcome association. The current results are perhaps the strongest evidence to date of the crucial role of the context.

The role of the PL seems to be constrained to excitatory, rather than inhibitory, instrumental responding. The present experiments, as well as others that examine the effect of PL inactivation on inhibitory responding, find no differences in inhibitory responding. For example, although Eddy et al. (2016) found that $A B A$ renewal was affected by PL inactivation, no differences were seen when rats were tested in Context $\mathrm{B}$, the context of extinction (see also Willcocks and McNally, 2013). In general, relapse effects that do not depend on memory for excitatory operant learning are not affected by PL inactivation, such as ABC renewal (Experiment 2a) or reacquisition (Corbit and Balleine, 2003).

Overall, the present results suggest that inactivation of the PL only affects responding when it is excitatory context-dependent responding tested in conditions that introduce a memory component (e.g., extinction, long delays between sample and test). This suggests that the PL may be crucially important for expression of excitatory responding that is learned during acquisition, as well as its return following extinction learning.

The renewal paradigm has long been thought to be an important analog to the return or relapse of behavior that is often seen following treatments for behavioral excesses, such as overeating, drug taking, or cigarette smoking. As such, understanding the mechanisms that contribute to it can provide important insight into how to reduce relapse and promote treatment effects. The current results provide one step toward this understanding, as well as further elucidate the role of the PL in operant conditioning more generally.

\section{References}

Baker AG, Steinwald H, Bouton ME (1991) Contextual conditioning and reinstatement of extinguished instrumental responding. Q J Exp Psychol Comp Physiol Psychol 43:199-218.

Balleine BW, Dickinson A (1998) Goal-directed instrumental action: contingency and incentive learning and their cortical substrates. Neuropharmacology 37:407-419. CrossRef Medline

Bossert JM, Stern AL, Theberge FR, Cifani C, Koya E, Hope BT, Shaham Y (2011) Ventral medial prefrontal cortex neuronal ensembles mediate context-induced relapse to heroin. Nat Neurosci 14:420-422. CrossRef Medline

Bossert JM, Marchant NJ, Calu DJ, Shaham Y (2013) The reinstatement model of drug relapse: recent neurobiological findings, emerging research topics, and translational research. Psychopharmacology 229:453-476. CrossRef Medline

Bouton ME, Todd TP, Vurbic D, Winterbauer NE (2011) Renewal after the extinction of free operant behavior. Learn Behav 39:57-67. x

Bouton ME, Todd TP, Leon SP (2014) Contextual control of discriminated operant behavior. J Exp Psychol Anim Behav Process 40:92-105. CrossRef Medline

Colwill RM, Rescorla RA (1985) Postconditioning devaluation of a reinforcer affects instrumental responding. J Exp Psychol Anim Behav Process 11:120-132. CrossRef

Corbit LH, Balleine BW (2003) The role of prelimbic cortex in instrumental conditioning. Behav Brain Res 146:145-157. CrossRef Medline

Coutureau E, Marchand AR, Di Scala G (2009) Goal-directed responding is sensitive to lesions to the prelimbic cortex or basolateral nucleus of the amygdala but not to their disconnection. Behav Neurosci 123:443-448. CrossRef Medline

Delatour B, Gisquet-Verrier P (1996) Prelimbic cortex specific lesions disrupt delayed-variable response tasks in the rat. Behav Neurosci 110:12821298. CrossRef Medline

Delatour B, Gisquet-Verrier P (1999) Lesions of the prelimbic-infralimbic cortices in rats do not disrupt response selection process but induce delaydependent deficits: evidence for a role in working memory? Behav Neurosci 113:941-955. CrossRef Medline

Delatour B, Gisquet-Verrier P (2000) Functional role of rat prelimbicinfralimbic cortices in spatial memory: evidence for their involvement in attention and behavioural flexibility. Behav Brain Res 109:113-128. CrossRef Medline

Eddy MC, Todd TP, Bouton ME, Green JT (2016) Medial prefrontal cortex involvement in the expression of extinction and $\mathrm{ABA}$ renewal of instrumental behavior for a food reinforcer. Neurobiol Learn Mem 128:33-39. CrossRef Medline

Fuchs RA, Evans KA, Ledford CC, Parker MP, Case JM, Mehta RH, See RE (2005) The role of the dorsomedial prefrontal cortex, basolateral amygdala, and dorsal hippocampus in contextual reinstatement of cocaine seeking in rats. Neuropsychopharmacology 30:296-309. CrossRef Medline 
Jonkman S, Mar AC, Dickinson A, Robbins TW, EverittBJ (2009) Therat prelimbic cortex mediates inhibitory response control but not the consolidation of instrumental learning. Behav Neurosci 123:875-885. CrossRef Medline

Kesner RP, Churchwell JC (2011) An analysis of rat prefrontal cortex in mediating executive function. Neurobiol Learn Mem 96:417-431. CrossRef Medline

Killcross S, Coutureau E (2003) Coordination of actions and habits in the medial prefrontal cortex of rats. Cereb Cortex 13:400-408. CrossRef Medline

Marquis JP, Killcross S, Haddon JE (2007) Inactivation of the prelimbic, but not infralimbic, prefrontal cortex impairs the contextual control of response conflict in rats. Eur J Neurosci 25:559-566. CrossRef Medline

Nakajima S, Tanaka S, Urushihara K, Imada H (2000) Renewal of extinguished lever-press responses upon return to the training context. Learn Motiv 31:416-431. CrossRef

Nakajima S, Urushihara K, Masaki T (2002) Renewal of operant performance formerly eliminated by omission or noncontingency training upon return to the acquisition context. Learn Motiv 33:510-525. CrossRef
Paxinos G, Watson C (2007) The rat brain in stereotaxic coordinates, Ed 6. Boston: Academic/Elsevier.

Ragozzino ME, Detrick S, Kesner RP (2002) The effects of prelimbic and infralimbic lesions on working memory for visual objects in rats. Neurobiol Learn Mem 77:29-43. CrossRef Medline

Thrailkill EA, Bouton ME (2015) Contextual control of instrumental actions and habits. J Exp Psychol Anim Learn Cogn 41:69-80. CrossRef Medline

Todd TP (2013) Mechanisms of renewal after the extinction of instrumental behavior. J Exp Psychol Anim Behav Process 39:193-207. CrossRef Medline

Trask S, Bouton ME (2014) Contextual control of operant behavior: evidence for hierarchical associations in instrumental learning. Learn Behav 42:281-288. CrossRef Medline

Willcocks AL, McNally GP (2013) The role of medial prefrontal cortex in extinction and reinstatement of alcohol-seeking in rats. Eur J Neurosci 37:259-268. CrossRef Medline 